\title{
An Empirical Approach to Correlating Thermo-Mechanical Fatigue Behaviour of a Polycrystalline Ni-Base Superalloy
}

\author{
Mark Whittaker ${ }^{1}$, Robert Lancaster ${ }^{1}{ }^{1}$, William Harrison ${ }^{1}$, Christopher Pretty ${ }^{1}$ and \\ Stephen Williams ${ }^{2}$
}

1 Materials Research Centre, College of Engineering, Swansea University, Singleton Park, Swansea SA2 8PP, UK; E-Mails: m.t.whittaker@swansea.ac.uk (M.W.); w.harrison@swansea.ac.uk (W.H.); 437761@ swansea.ac.uk (C.P.)

2 Rolls-Royce plc, Elton Road, Derby DE24 8BJ, UK; E-Mail: steve.williams@ rolls-royce.com

* Author to whom correspondence should be addressed: E-Mail: r.j.lancaster@swansea.ac.uk; Tel.: +44-179-260-2061; Fax: +44-179-229-5693.

Received: 31 October 2013; in revised form: 7 November 2013 / Accepted: 12 November 2013 / Published: 15 November 2013

\begin{abstract}
Assessment of thermo-mechanical fatigue behaviour of the polycrystalline nickel alloy RR1000 reveals a significant effect of phase angle on fatigue life. The current paper explores two scenarios: the first where the mechanical strain range is held constant and comparisons of the fatigue life are made for different phase angle tests; and secondly, the difference between the behaviour of In-phase (IP) and $-180^{\circ}$ Out-Of-Phase (OOP) tests over a variety of applied strain ranges. It is shown that different lifing approaches are currently required for the two scenarios, with a mean stress based approach being more applicable in the first case, whereas a Basquin-type model proves more applicable in the second. However, it is also demonstrated that the crack propagation phase should also be considered in these types of tests for high strain ranges and projects that future modelling approaches should attempt to unify mean stress, stress range and a crack propagation phase.
\end{abstract}

Keywords: thermo-mechanical fatigue; nickel superalloys; modelling; crack propagation 


\section{Introduction}

It is widely acknowledged that thermo-mechanical fatigue (TMF) is one of the most significant challenges facing the gas turbine sector as designers strive to meet the demanding emissions and fuel burn ACARE (Advisory Council for Aeronautics Research and Innovation in Europe) "Flightpath 2050" targets. Increasing operating temperatures to improve cycle efficiency, in conjunction with weight reduction strategies that include thinner disc rims, have led not only to more aggressive TMF cycles, but also to the requirement for assessment of the behaviour of components for which TMF was not previously considered significant.

TMF testing however is difficult, expensive and time consuming and there is a wide range of variables that require consideration. Such variables include peak cycle temperature, temperature cycle range, applied strain range, $R$ ratio, phase angle (between thermal and mechanical strains), waveform and applied strain rate. Accordingly, a data generation programme that encompasses all of these factors is expensive and unrealistic. Therefore there is a clear requirement for a robust predictive model which can provide accurate extrapolation from a known set of results. Furthermore, it is desirable that the model, if possible, utilise isothermal data in order to further simplify the input data requirements. However, this is obviously a significant extension of models which predict only isothermal data and it is necessary to investigate whether such models are compromised by the damage mechanisms involved in TMF.

This paper focuses on modelling the effects of phase angle on TMF life in the polycrystalline nickel alloy RR1000. RR1000 is commonly produced through a powder metallurgy process, which is widely anticipated to play a significant role in upcoming engines as a turbine disc material. However studies into the TMF behaviour of the alloy have been extremely limited and TMF lifing of many alloys tends to follow the approach of attempting extrapolation from isothermal data and particularly assuming isothermal data at the peak cycle temperature to be a worst case scenario. Within the gas turbine, however, this approach may be extremely limited due to the wide range of phase angles possible and the marked effect of a TMF cycle.

The phase angle as defined for a TMF test is the shift which occurs between the mechanical strain and the temperature applied to the material. If the mechanical strains and temperature increase/decrease in proportion with each other, the cycle is considered to be In-Phase (IP). If one always increases as the other decreases, the cycle is Out-Of-Phase (OOP) or more accurately, $-180^{\circ}$ Out-Of-Phase. Clearly there are also an infinite number of potential phase angles which could be applied between these two extremes and furthermore an added complication is that the cycle can take one of two directions around the strain-temperature loop, either clockwise (CW) or anticlockwise (ACW), as shown in Figure 1.

Laboratory simulations of TMF testing are often, by necessity, idealised versions of in service cycles. It is impossible to undertake a TMF testing programme which will encompass all of the possible variations which may occur in service. However, the range of phase angles that may occur in component TMF cycles will be covered by tests performed for a number of standard phase angles together with modelling work that allows the results to be interpolated between phase angles and, ideally, to different maximum and minimum cycle temperatures. This will give designers the ability to predict lives for all component loading cycles. As such it is critical that any model which is sought to 
describe TMF behaviour is capable of interpolation across phase angles, and if at all possible different maximum/minimum temperatures, in order to allow designers the capacity for accurate life prediction of critical parts.

Figure 1. Phase angles $(\phi)$ and directions (clockwise $(\mathrm{CW})$ or anticlockwise (ACW)).

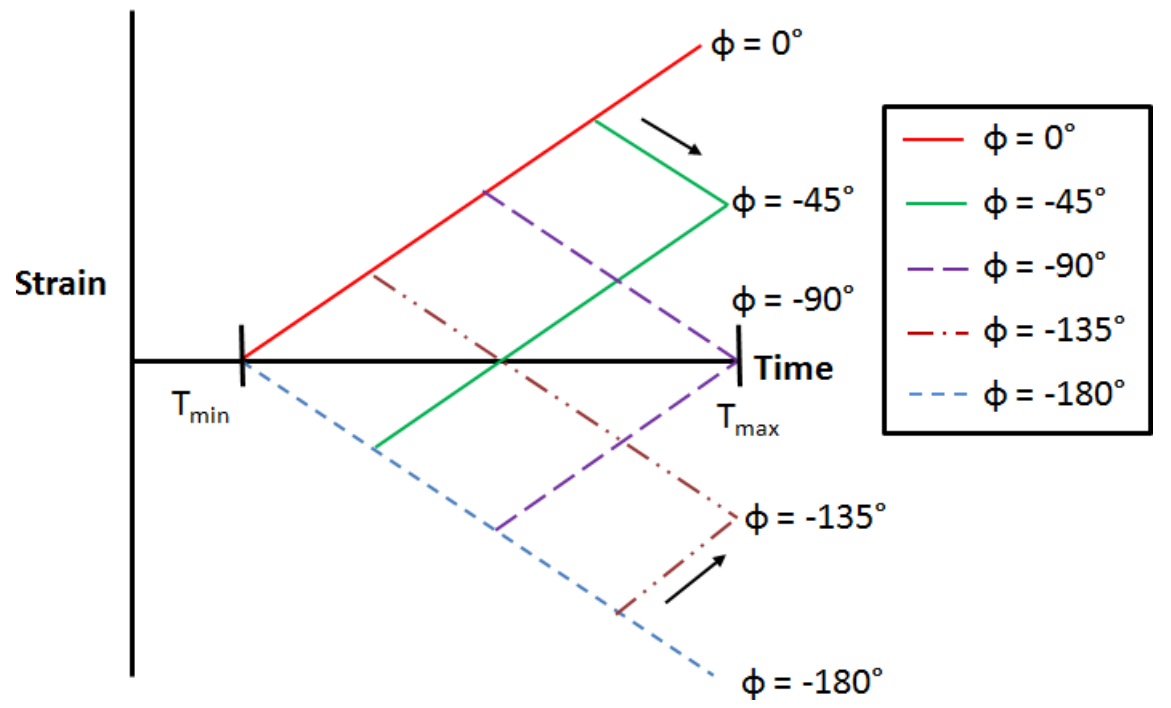

Previous attempts to model TMF behaviour in the literature [1-5], especially in nickel based superalloys for disc applications, are quite limited. They do however, fall broadly into two categories; the first assumes that under strain control loading a significant fraction of the TMF life is spent in crack initiation and as such traditional approaches such as strain-energy partitioning, Manson-Coffin [6] etc., can be applied [7-10]. Conversely, the second approach assumes that cracks form very early in the test, possibly even on the first cycle [11-13]. On this basis the fatigue life of the specimen will be dominated by crack propagation. Each type of approach has been shown to have its own benefits and drawbacks and it is also clear that the most appropriate method will be material and temperature dependent.

\section{Experimental Methods}

The TMF facility utilised at Swansea for the current testing consisted of an ESH $100 \mathrm{kN} / 400 \mathrm{Nm}$ tension-torsion capable servo-hydraulic machine. Extension was controlled by a $12 \mathrm{~mm}$ gauge length MTS high temperature extensometer. Application of the thermal cycle was achieved through a water cooled radio-frequency RF induction coil, with internal and external jets of forced air providing cooling. The temperature was measured and controlled by an IMPAC IP10 optical pyrometer connected to a two way control system. Tubular hollow tests specimens of nominal external diameter $10 \mathrm{~mm}$ and internal diameter $8 \mathrm{~mm}$ were utilised for testing, as shown in Figure 2. The temperature distribution across the test specimen was shown to conform to the guidelines defined in ISO 12111:2011 [14], i.e., the linear temperature distribution within the extensometer arms remains less than $\pm 10{ }^{\circ} \mathrm{C}$ at peak temperature and $\pm 6{ }^{\circ} \mathrm{C}$ at minimum temperature, and the radial temperature distribution remains within $5 \%$ of the required temperature. In accordance to the TMF Code of 
Practice (TMF COP) [15], standard pre-requisite tests including modulus checks, thermal compensation definitions and zero stress trials were also performed to ensure test viability.

As described previously, the material chosen for the study was the polycrystalline nickel alloy RR1000. As a heavily $\gamma^{\prime}$ stabilised nickel alloy, primary ( 5 $\mu \mathrm{m}$ diameter $)$ and secondary $\gamma^{\prime}(\sim 0.2 \mu \mathrm{m}$ diameter) precipitates were easily visible by Scanning Electron Microscopic (SEM) analysis, as shown in Figure 3. Finer $\gamma^{\prime}$ precipitates have previously been observed in the material through the use of Field Emission Gun (FEG) SEMs. The grain size of the material was approximately $8 \mu \mathrm{m}$ and was determined by a standard mean linear intercept method. This resulted in on average, greater than 500,000 grains being sampled across the cross section of each test specimen.

Figure 2. Specimen diagram of test pieces used in this research.

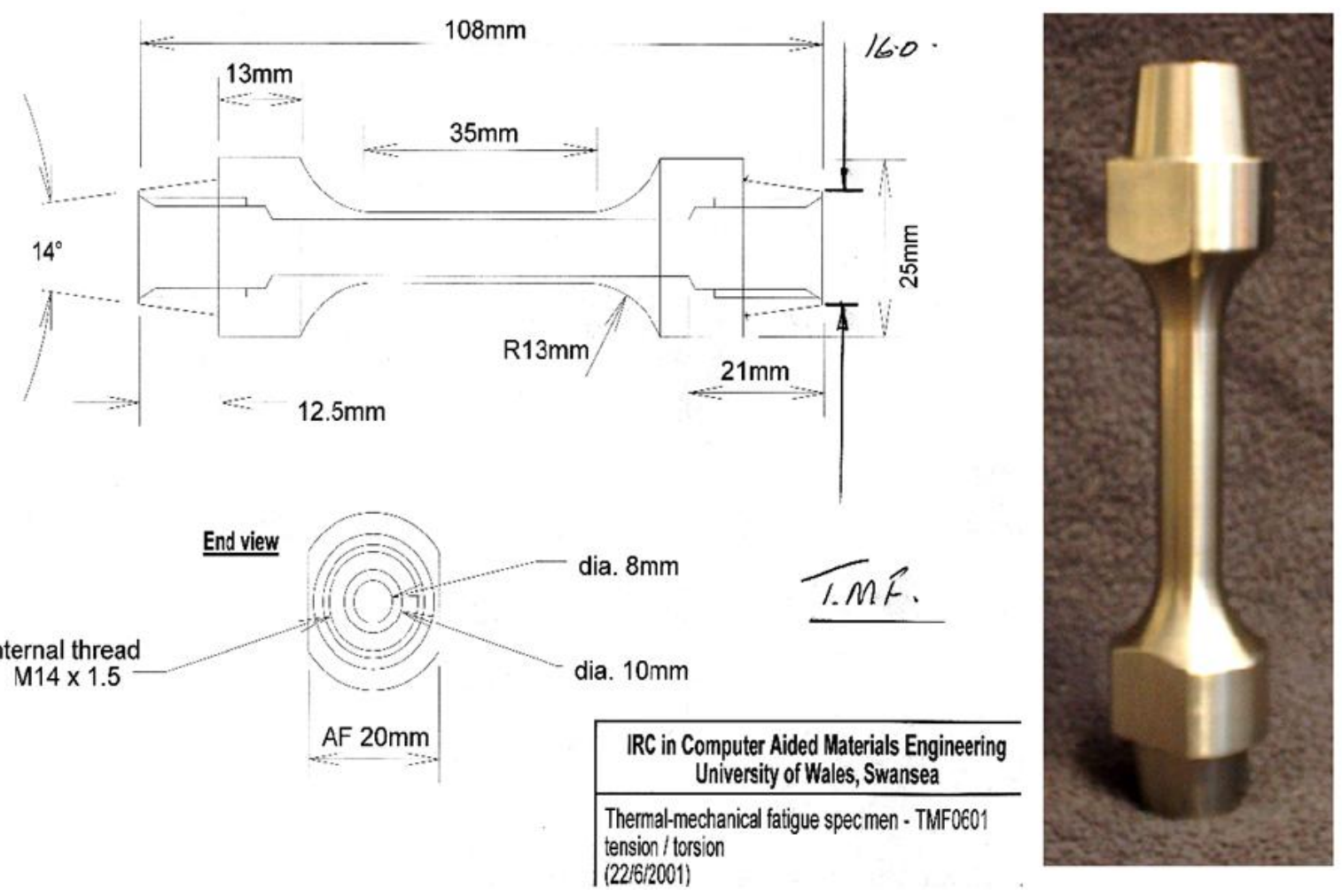

Figure 3. Microstructure of RR1000.

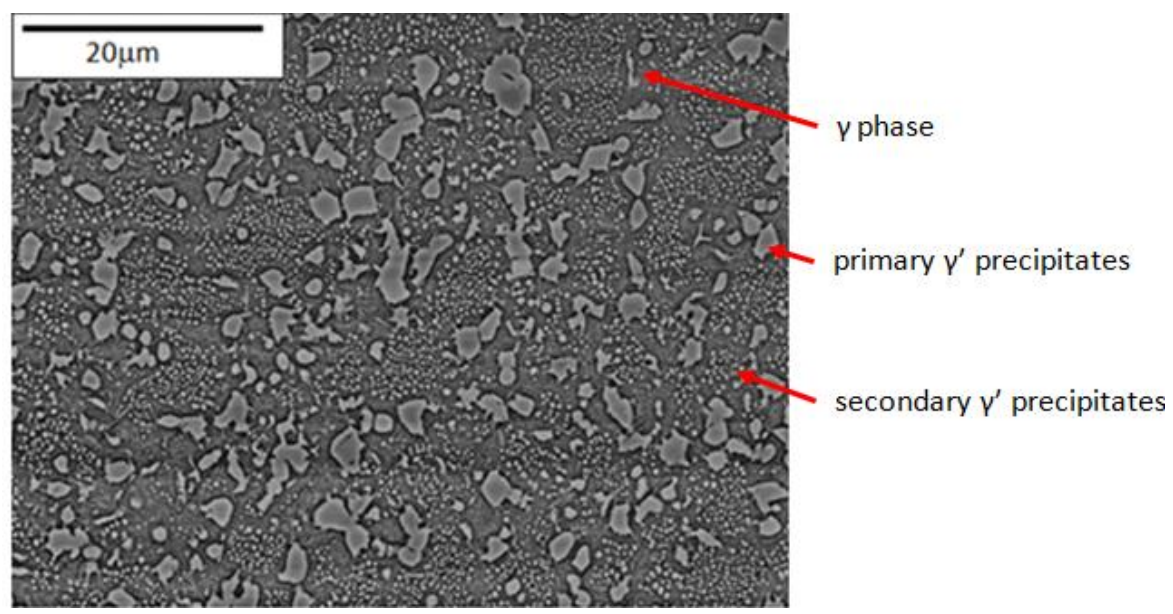


The temperature cycle used during the testing was (300-700-300) ${ }^{\circ} \mathrm{C}$, linearly, in $30 \mathrm{~s}$, which was thought to be representative of a typical in-service TMF cycle for a turbine disc. Clearly concerns have previously been raised regarding the use of pyrometry for this form of testing, since changes can occur in the material's emissitivity if it is subjected to significant periods of time at elevated temperature, causing oxidation. In order to overcome this issue, the emissivity of the material was characterised as a function of time and temperature. It was shown that at $700{ }^{\circ} \mathrm{C}$ the emissivity of RR1000 reaches a stable value after $8 \mathrm{~h}$. As such, specimens were pre-oxidised in a furnace for $8 \mathrm{~h}$ to create a stable oxide layer prior to the commencement of any TMF experiments. No further changes in emissivity were noted, and subsequently accurate temperature control was achieved throughout the test.

In order to evaluate the effect of phase angle on the TMF life of RR1000, tests were performed at $\phi=0,-45(\mathrm{CW}),-90(\mathrm{CW}+\mathrm{ACW}),-135(\mathrm{ACW})$, and $-180^{\circ}$ as shown in Figure 1 . Tests were performed using a constant mechanical strain range of $1 \%$, resulting in strain $\mathrm{R}$ ratios varying between 0 and $-\infty$ depending on the phase angle $\phi$. Further tests were performed under IP and $-180^{\circ}$ OOP loading conditions in which the applied mechanical strain range was varied between $0.8 \%$ and $1.4 \%$ in order to investigate the effect of this property on TMF life. Failure was defined as the number of cycles to achieve a $10 \%$ drop in stress from the stabilised peak stress value at which point on average, a semi-circular crack of length $0.7 \mathrm{~mm}$ was recognisable in the sample $[14,15]$

\section{Results}

In order to derive a model that accurately describes TMF behaviour, it is of course critical that the data produced by the experiments is of the highest quality possible. In the case of the current work the quality of the test data can be easily determined from the appearance of the mechanical strain-stress hysteresis loops. During the experiments the data returned appears to be of a high quality, allowing for consistent interpretation. Figure 4 provides examples of the loops obtained from both IP and $-180^{\circ}$ OOP tests with strain ranges of $1.4 \%$. As would be expected in TMF loops, yield is not particularly well defined due to the changing modulus values as temperature increases in the initial part of the cycle. The change in the minimum stress (whilst the maximum stress remains constant) in the IP loop is also indicative of the competing issues of creep and plastic strain occurring at the cycle strain and temperature extremes. Some cyclic hardening of the plastic behaviour can also be seen to occur between the first and stabilised loading cycles (Figure 4a). Similar effects can also be observed in compression in the $-180^{\circ}$ OOP loop (Figure $4 \mathrm{~b}$ ).

With the quality of the stress-strain loops established, confidence can be placed in the fatigue lives of the tested specimens. The data from Table 1 for the range of tests is plotted in Figure 5. In order to relate isothermal behaviour to TMF, the lives of the tests are normalised by the fatigue life of an isothermal fatigue test on the material at $R=0, \Delta \varepsilon=1 \%$ at peak cycle temperature $\left(700{ }^{\circ} \mathrm{C}\right)$, and therefore a positive or negative effect of the TMF cycle can be easily demonstrated. Essentially, two studies were undertaken, as described previously. In Figure 5 tests are conducted at a range of phase angles as described by Figure 1, whilst maintaining a constant applied mechanical strain range, $\Delta \varepsilon=1 \%$. 
Figure 4. 1 st and stabilised stress-strain hysteresis loops for (a) In-Phase (IP) test, $\Delta \varepsilon=1.4 \%$;

(b) Out-Of-Phase (OOP) test, $\Delta \varepsilon=1.4 \%$.

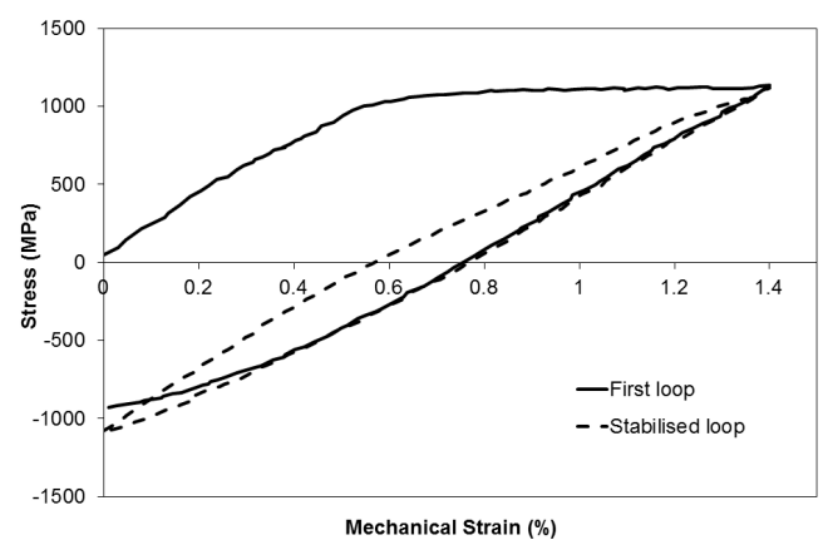

(a)

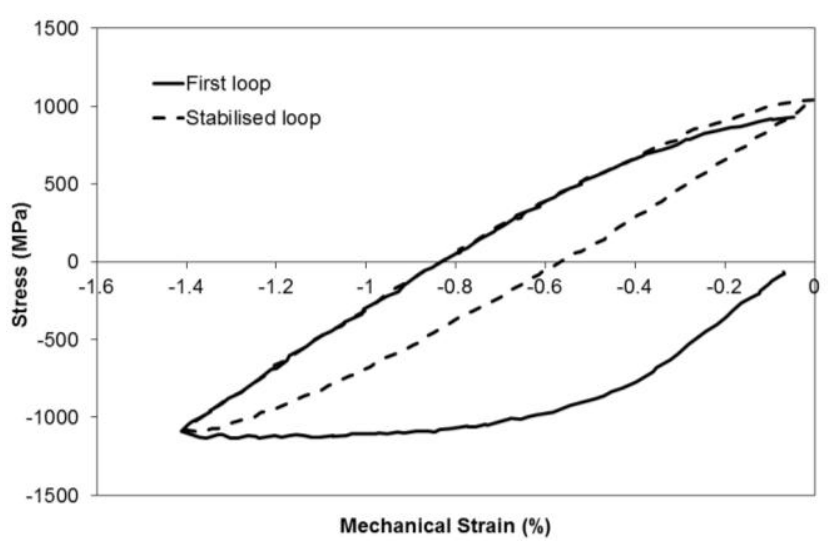

(b)

Table 1. Experimental Data.

\begin{tabular}{|c|c|c|c|c|c|c|c|c|}
\hline $\begin{array}{c}\text { Phase } \\
\text { angle }\left({ }^{\circ}\right)\end{array}$ & $\begin{array}{c}\text { Max } \\
\text { Strain (\%) }\end{array}$ & $\begin{array}{c}\text { Min Strain } \\
(\%)\end{array}$ & $\begin{array}{c}\text { Strain } \\
\text { Range (\%) }\end{array}$ & $\begin{array}{c}\text { Stabilised Max } \\
\text { Stress (MPa) }\end{array}$ & $\begin{array}{l}\text { Stabilised Min } \\
\text { Stress (MPa) }\end{array}$ & $\begin{array}{c}\text { Stabilised Stress } \\
\text { Range (MPa) }\end{array}$ & Direction & Normalised $N_{\mathrm{f}}$ \\
\hline 0 & 0.98 & -0.03 & 1.01 & 978 & -763 & 1741 & $\mathrm{CW}$ & 0.49 \\
\hline 0 & 1.2 & 0 & 1.2 & 925 & -1159 & 2084 & $\mathrm{CW}$ & 0.271 \\
\hline 0 & 1.4 & -0.01 & 1.41 & 1125 & -1082 & 2207 & $\mathrm{CW}$ & 0.156 \\
\hline 0 & 0.79 & -0.02 & 0.81 & 933 & -631 & 1564 & $\mathrm{CW}$ & 12.5 Unbroken \\
\hline 0 & 0.89 & -0.02 & 0.91 & 1019 & -681 & 1700 & $\mathrm{CW}$ & 0.656 \\
\hline-45 & 0.74 & -0.3 & 1.04 & 1045 & -849 & 1894 & $\mathrm{CW}$ & 0.75 \\
\hline-90 & 0.48 & -0.52 & 1 & 857 & -925 & 1782 & $\mathrm{ACW}$ & 1.198 \\
\hline-90 & 0.52 & -0.49 & 1.01 & 945 & -895 & 1840 & $\mathrm{CW}$ & 1.243 \\
\hline-135 & 0.27 & -0.74 & 1.01 & 826 & -993 & 1819 & $\mathrm{ACW}$ & 1.385 \\
\hline-135 & 0.32 & -0.7 & 1.02 & 741 & -1049 & 1790 & $\mathrm{ACW}$ & 1.219 \\
\hline-180 & 0.05 & -1.05 & 1.1 & 865 & -982 & 1847 & $\mathrm{ACW}$ & 1.12 \\
\hline-180 & -0.01 & -1.22 & 1.21 & 926 & -1047 & 1973 & $\mathrm{ACW}$ & 0.729 \\
\hline-180 & 0 & -1.41 & 1.41 & 1105 & -1070 & 2175 & $\mathrm{ACW}$ & 0.13 \\
\hline-180 & 0 & -0.81 & 0.81 & 630 & -864 & 1494 & $\mathrm{ACW}$ & 8.703 \\
\hline
\end{tabular}

The conditions of these tests offer an interesting opportunity for comparison, since although the $R$ ratio varies from test to test, the selected phase angles provide identical strain-temperature paths for portions of each cycle. It is clear that phase angle significantly affects TMF life, with a gradual decrease in life as the phase angle increases from $-180^{\circ}$ to $0^{\circ}$, as shown in Figure 5. Table 1 describes further IP and OOP tests for strain ranges between $0.8 \%$ and $1.4 \%$. These results form the basis for a predictive model for TMF behaviour described within this paper. 
Figure 5. Phase angle $(\phi) v s$. number of thermo-mechanical fatigue (TMF) cycles to failure $\left(N_{\mathrm{f}}\right)$ for constant strain range $(\Delta \varepsilon)$ of $1 \%$.

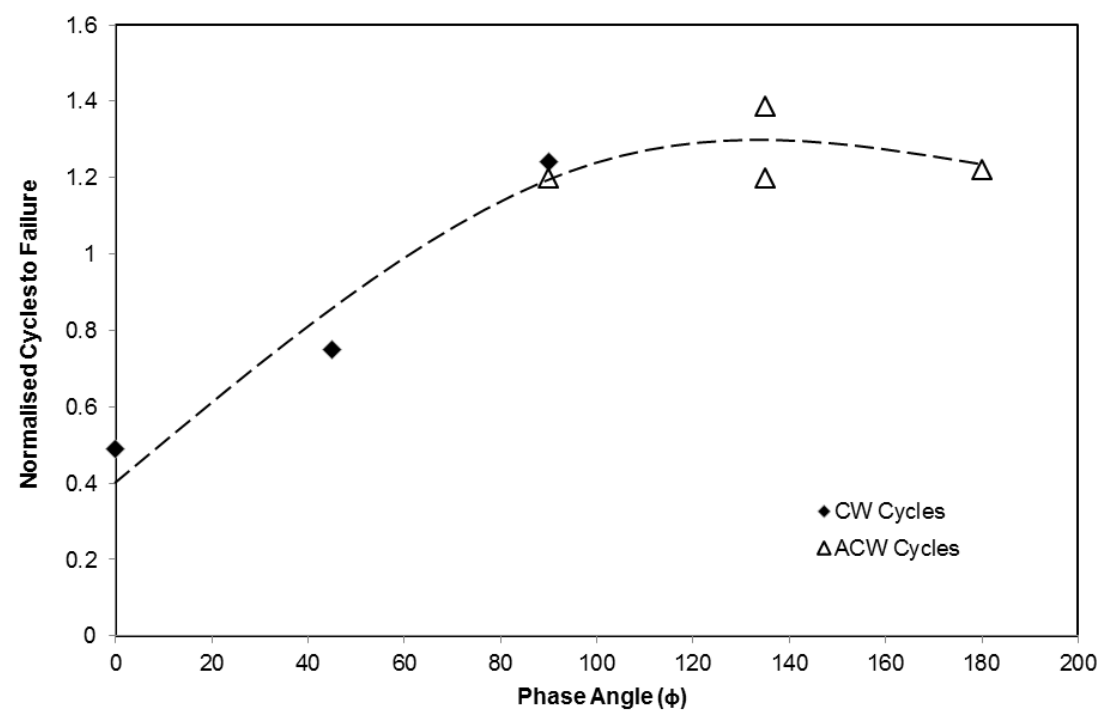

\section{Discussion}

Based on the wide range of variables which occur during a TMF test it is clear that a lifing approach capable of holistic data fitting may not be possible. However, an objective of the current work is to assess possible lifing strategies in order to provide a basis for a more widespread approach. As such, the discussion will focus on the advantages and drawbacks of methods based around mean stress, stabilised stress range and crack propagation life. Based on the results produced within this work, no difference was seen between specimens loaded in a clockwise (CW) or anticlockwise (ACW) direction around the strain-temperature loop, for $-90^{\circ}$ OOP tests. As such the approaches here are simplified by not considering directionality in these tests.

One relatively straightforward approach to ordering TMF results can be made by simply considering the stabilised mean stress achieved during the test, with the expectation being that an increased mean stress for a constant stress range will lead to a reduction in fatigue life. Due to the non-isothermal nature of the TMF test, stress-strain hysteresis loops can vary dramatically in shape for different phase angles, leading to significant changes in mean stress. The most common cause of this is the alternating contributions between creep strain and plasticity, typical in the TMF cycle. By considering an IP cycle, in which the strain and temperature increase in proportion, it can be appreciated that yield will occur within the material and dislocations will be generated within the grains, resulting in plastic deformation. However, at higher temperatures creep deformation becomes more significant in the alloy, which acts to lower the applied stress through stress relaxation, since the test is under strain control. Under an IP TMF cycle, with creep therefore dominating at the peak strain, this stress relaxation acts to decrease the mean stress throughout the test. Conversely in a $-180^{\circ}$ OOP test, creep is most prevalent at the minimum strain, and therefore acts to increase the mean stress, as can be observed in Figure 4.

Clearly for a series of tests performed at different strain ranges, this approach is overly simplistic in the presence of varying peak stresses and stress ranges. For example a $-180^{\circ}$ OOP test with a high strain range would not be expected to show a longer fatigue life than an IP test with a low strain range, 
simply because it has a lower value of mean stress. However, for the current evaluation of the effect of phase angle on fatigue life, the method can be instructive for a series of experiments where a constant strain range is utilised.

Following initial plasticity on loading, as demonstrated in Figure 4, similar stress ranges occur across the range of phase angles, enabling the effect of mean stress to be more clearly evaluated. Figure 6 shows a comparison of mean stress against normalised cycles to failure for the range of tests performed at $\Delta \varepsilon_{\text {mech }}=1 \%$. The data is normalised by the number of fatigue cycles to failure for an isothermal test, performed at the peak temperature $\left(700^{\circ} \mathrm{C}\right)$ of the TMF cycle and clearly demonstrates the effects of mean stress with values $<1$ showing a detriment to life, and values $>1$ showing an extension of life. With an $R^{2}$ value of 0.81 it is clear that a relationship exists and that the TMF life is strongly dependent on mean stress. However, it should again be emphasised that this relationship exists only for specimens with a comparable stabilised stress range, brought about in these experiments by the application of a constant applied strain range of $1 \%$ throughout the tests. In the case of extrapolation to a varying applied strain range (and hence stabilised stress range) the relationship holds little value because of the tendency of stabilised stress range to become the dominant factor.

Figure 6. Mean stress $v s$. cycles to failure for varying phase angle tests at $\Delta \varepsilon$ of $1 \%$.

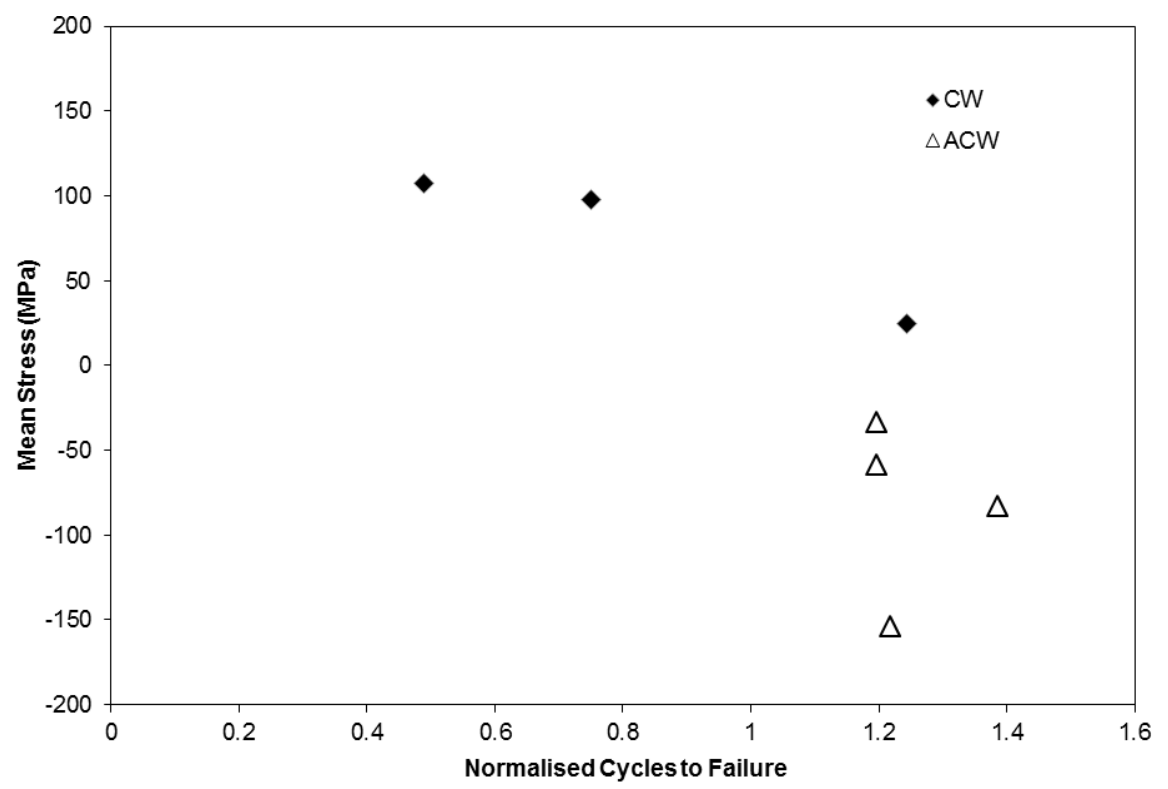

Typically, more holistic fatigue lifing approaches will be either strain range $[6,7,16]$ or stress range [17] based. In the current work a strong correlation between stabilised stress range and TMF life in the IP and $-180^{\circ} \mathrm{OOP}$ experiments indicates that a Basquin [17] type approach could potentially form the basis of a model which is capable of TMF life prediction and extrapolation across a range of phase angles.

As mentioned previously however, TMF experiments are costly, difficult and time consuming and it is preferable that predictions can be made based on isothermal data. However, as indicated in the literature [18], simple "worst case scenario" and generalised fatigue life reduction factors fail to capture the complexities of the effect of phase angle, potentially leading to overly conservative life predictions or even premature component failures. In the present work, efforts have been made to effect a creep and strain controlled fatigue prediction based on TMF hysteresis loops which have been derived from a database of isothermal RR1000 test results. SC03 is a non-linear finite element analysis 
program which utilises the Mroz multilayer plasticity model to predict cyclic deformation based on stress-strain data obtained from isothermal testing [19]. The wide range of input data used to produce the deformation model for RR1000 provides confidence in its ability to predict conditions outside of the envelope of this original data. It also allows for an iterative approach over both temperature and strain conditions by which the replication of TMF stress-strain hysteresis loops can be achieved.

Examples of loops produced by SC03 are shown in Figure 7 and compared with experimental data generated under TMF loading conditions. It can be seen that peak stress, minimum stress and loop shape are all captured well by the model, providing confidence in its ability to extrapolate to alternative phase angles and produce a model which can provide TMF predictions over a wide range of phase angles when used in association with predicted stresses from SC03 to estimate TMF test failure lives. As described earlier a strong relationship exists between the stabilised stress range and the fatigue life, and as such Basquin's law [17] has been applied to derive a predictive equation for the experiments.

Figure 7. Modelling of TMF loops (a) IP $\Delta \varepsilon$ of $0.8 \%$; (b) $-180^{\circ}$ OOP $\Delta \varepsilon$ of $1.2 \%$.

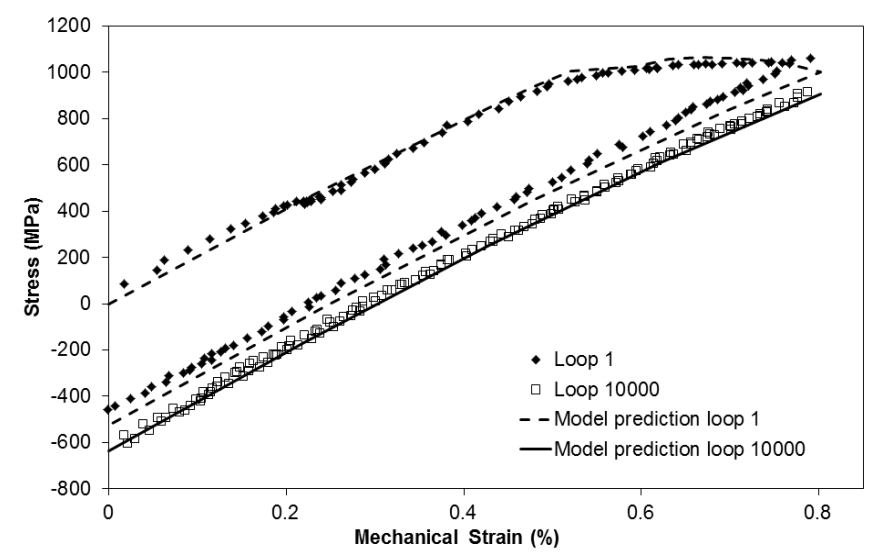

(a)

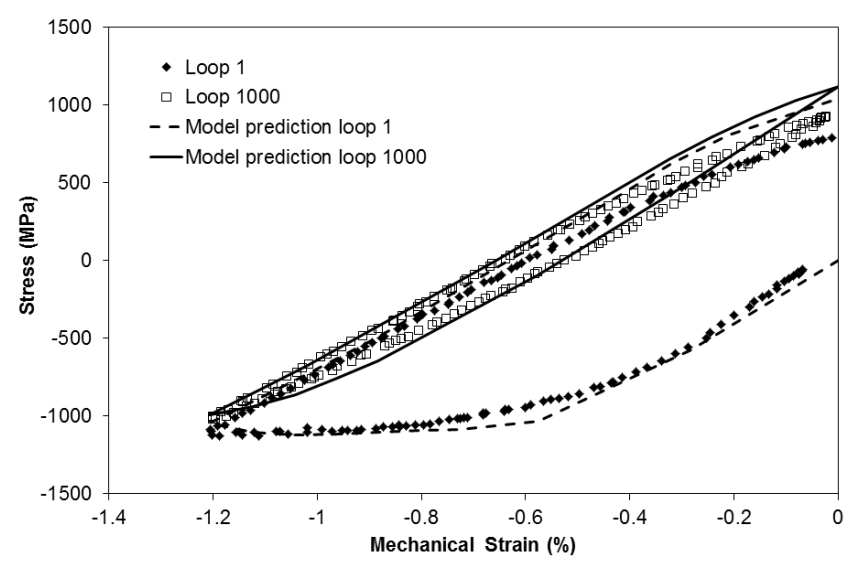

(b)

In order to prove the effectiveness of the technique, the predictive equation has been derived based purely on IP experiments. Stress-strain hysteresis loops were then produced for each of the remaining tested conditions $\left(4 \times-180^{\circ} \mathrm{OOP},-45^{\circ} \mathrm{CW},-90^{\circ} \mathrm{CW},-90^{\circ} \mathrm{ACW},-135^{\circ} \mathrm{ACW}\right)$ and predictions made of TMF life using the Basquin approach [17]. The equation takes the form

$$
\frac{\Delta \sigma}{2}=\sigma_{f}^{\prime}\left(2 N_{f}\right)^{b}
$$

Where $\Delta \sigma$ is the stress range and $N_{\mathrm{f}}$ is the number of TMF cycles to failure again normalised by the fatigue life of an isothermal test at $\Delta \varepsilon=1 \%$ at peak cycle temperature $\left(700{ }^{\circ} \mathrm{C}\right)$, for consistency. The constants were optimised at $\sigma_{\mathrm{f}}^{\prime}=989.5, \mathrm{~b}=-0.0818$.

Figure 8 shows the predictions of phase angles between $0^{\circ}$ and $-180^{\circ}$ using the above equation. It is clear that reasonable predictions are obtained for all of the data. If anything the method has the tendency to slightly underpredict the results. This may be a result of differences in the crack propagation life of the IP and $-180^{\circ}$ OOP tests brought about by the difference in temperature at peak stress. Furthermore it could be argued that when considering the range of phase angle tests performed at $\Delta \varepsilon_{\text {mech }}=1 \%$ the method fails to capture differences between the phase angles, and certainly does not 
separate the results in the manner in which the mean stress approach did. It should be noted however, that only a factor of 2 difference is observed in the failure life of these experiments, and for this reason it is difficult to draw strong conclusions. Furthermore, it is also necessary to consider that due to a combination of limited creep strain accumulation at the peak cycle temperature, the accumulation of significant mean stresses does not occur in the current tests. Future approaches for higher peak cycle temperatures may be well advised to consider either an adaptation of the Basquin approach to also consider mean stress, or the application of an approach based on both stress range and mean stress.

Figure 8. Basquin fit predictions of TMF behavior.

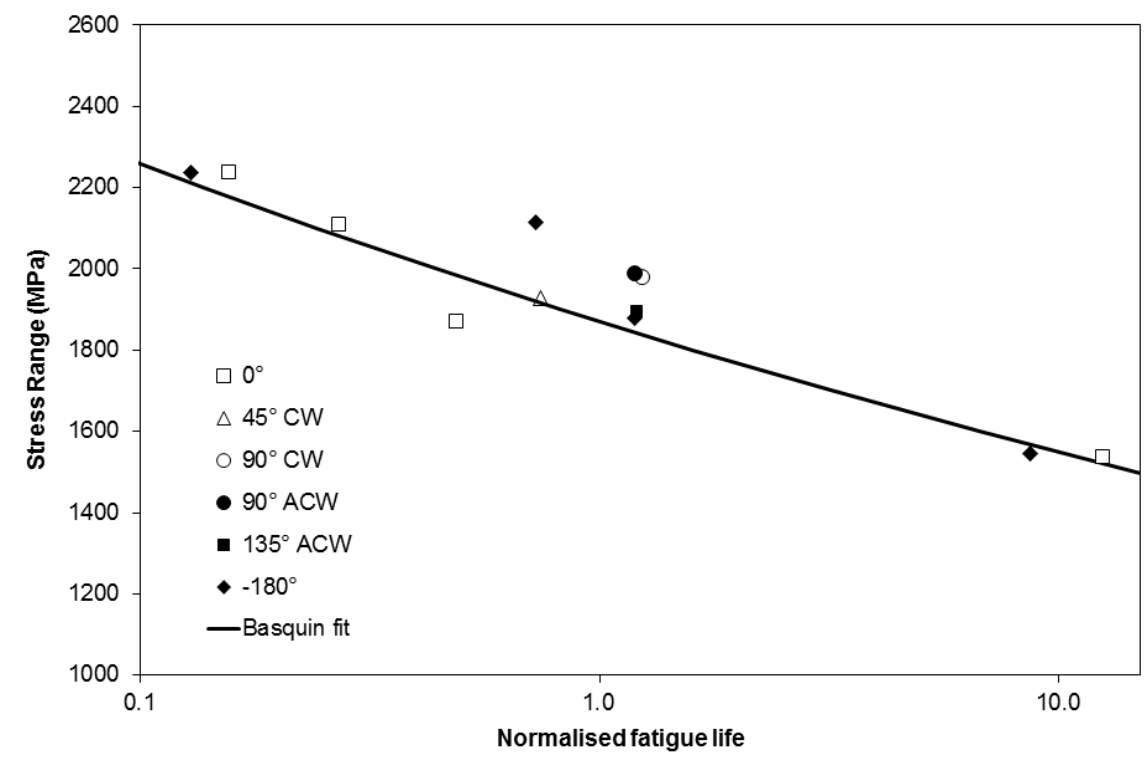

Both of the approaches detailed so far consider only a total life philosophy. Previous work [20] has indicated that the ordering of IP, OOP and IF (Isothermal Fatigue) tests may prove difficult for short TMF life experiments when using only methods such as Basquin [17] or Manson-Coffin [6,7]. Indeed the ordering of these test results may prove counterintuitive to the ordering seen for longer life experiments. As such it is useful to further investigate the length of the crack propagation phase within the current series of tests, so that the ratio of crack initiation/propagation can be considered.

Although a facility for investigating TMF crack propagation rates is under development at Swansea University, information regarding these rates is extremely limited in the published literature [21-24]. A fractographic study was undertaken of the tested specimens and observations have revealed extensive striations in the $-180^{\circ}$ OOP specimens, Figure 9. Past observations in the literature have indicated that striation counting in nickel based superalloys is a reliable means of estimation of crack propagation rates $[25,26]$. As such it was possible to identify a crack initiation site and count striations until failure occurred in the specimen by ductile overload.

The number of striations were recorded for two $-180^{\circ}$ OOP specimens $\left(\Delta \varepsilon_{\text {mech }}=1.2 \%\right.$ and $\left.1.4 \%\right)$ and one $-90^{\circ}$ OOP ACW specimen $\left(\Delta \varepsilon_{\text {mech }}=1 \%\right)$. The fractured IP testpieces were examined for striations, but determination was found to be difficult due to the clear interactions of other high temperature damage mechanisms (i.e., creep and environmental damage) at peak stress in these experiments. It should be noted that a comprehensive review of damage mechanisms occurring under TMF loading in nickel based superalloys is available in the open literature [27]. However, in order to 
simplify the lifing approaches applied here, time dependent damage mechanisms (i.e., creep and environmental damage) are considered as a single entity.

Observations show that significant fractions of these experiments are spent in crack propagation, since the striation count approaches the total specimen life. Indeed all three specimens showed striations that accounted for $>80 \%$ of the total fatigue life. Furthermore if the striation counts are believed to be accurate, the initiation phase diminishes with increasing strain until, allowing for minor counting errors, the material cracks on the first cycle in the $\Delta \varepsilon_{\text {mech }}=1.4 \%,-180^{\circ}$ OOP test, and spends the remainder of its life in propagation.

Figure 9. Fracture surface of RR1000 from an OOP $180^{\circ}$ anti-clockwise test (TMF013), with $\Delta \varepsilon_{\text {mech }}=1.4 \%$ resulting in a life of $N_{\mathrm{f}}=312$.

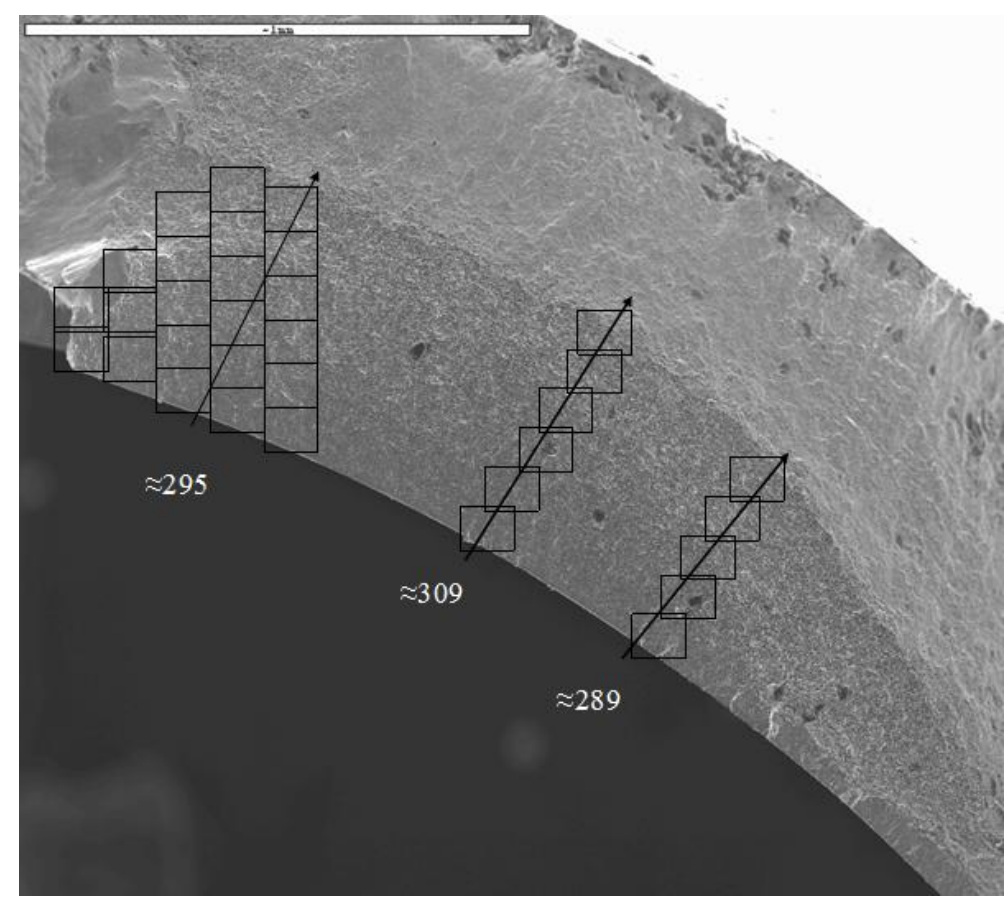

Whilst the previous approach based on Basquin's method shows a reasonable fit, it is unlikely that a single fit would prove adequate if more results with TMF lives $>1000$ cycles were generated. With crack initiation likely to dominate life, the crack propagation phase would become negligible in the determination of a predictive equation, and hence the prediction would most likely underpredict some of the short term test data observed in the current work. As such, in order to produce a total life prediction methodology, it is clear that there is a requirement for an approach which can also account for crack propagation, rather than assuming total life to be a result of a long crack initiation period followed by rapid crack growth to failure. An existing database of isothermal data in RR1000 can again be accessed for this purpose.

In order to derive a prediction for the propagation rates of TMF experiments a model has been produced based on the aforementioned isothermal data. Provided the strain controlled TMF cycle is accurately described, in terms of time, temperature, peak stabilised stress, strain range and phase angle, these simple variables can be used as inputs for the model. Furthermore the aspect ratio of the crack is required in order to calculate the geometry factor, $Y$, such that $\Delta K=Y \Delta \sigma \sqrt{\pi a}$ ( $\Delta K$ is stress intensity 
range, $\Delta \sigma$ is stabilised stress range, $a$ is crack length). Based on typical crack shapes observed in the current specimens, the variation of $\Delta K$ was estimated with increasing crack depth, a. In order to achieve this, a simplified approach was utilised where the hollow cylindrical test piece was approximated by a rectangular geometry. Analysis of the relationship between crack size and test piece geometry meant that this approximation was deemed reasonable. Due to the relatively long gauge length of the specimen, no significant effects of boundary conditions influenced the calculations. A stress intensity profile for a crack growing in the current hollow cylindrical specimens can be seen in Figure 10.

Time dependent and time independent crack growth rates are then calculated, so that the contribution from the two damage mechanisms at high temperature can be understood. The time dependent crack growth term, based on an Arrhenius type expression, is of the form:

$$
\frac{d a}{d t}=A^{*} e^{-Q / R T} K^{n}
$$

Where $d a / d t$ is the time dependent rate of crack growth, $A^{*}$ and $n$ are material fitting constants, $Q$ is the activation energy for time dependent crack growth $\left(265 \mathrm{~kJ} / \mathrm{mol}^{-1}\right), R$ is the gas constant, $T$ is temperature and $K$ is the stress intensity factor.

The time dependent crack growth rate is integrated through the whole loading cycle and therefore implicitly includes any minor cycle effects. Time dependent crack growth will occur when both high temperatures and high tensile stresses are present, such as in an IP test.

Figure 10. Stress intensity $(K) v s$. crack length $(a)$ for a semi-elliptical crack as shown in Figure 9.

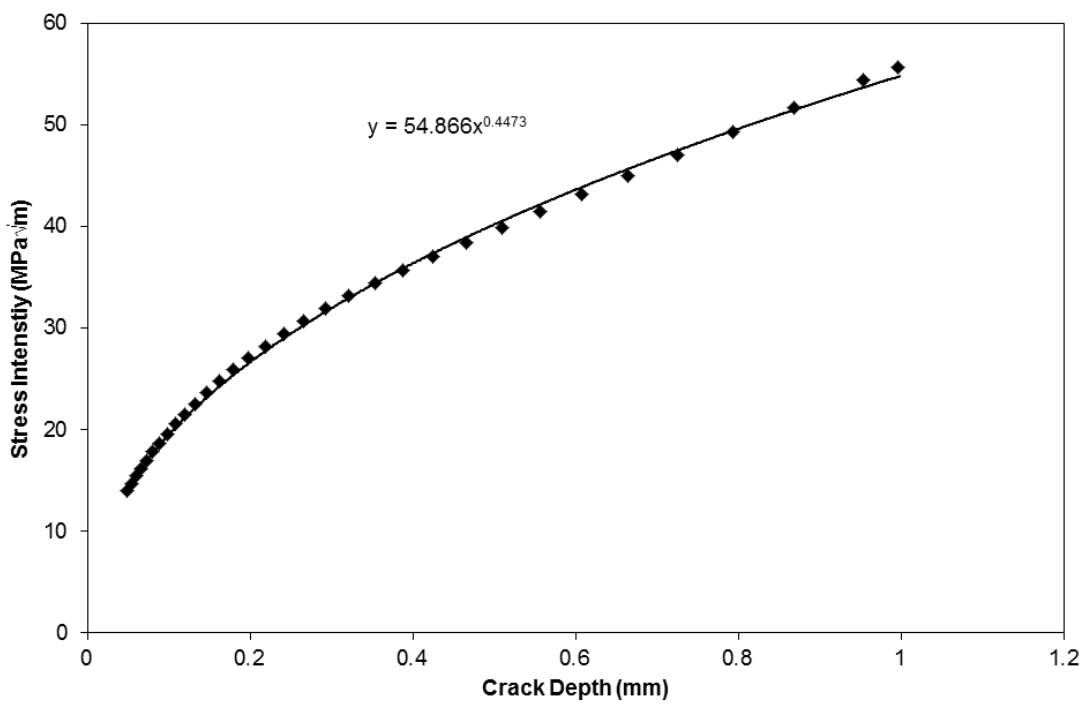

The model identifies minor cycles based on stress turning points and accounts for them in the time independent crack growth rate calculation through an exchange rate term. The exchange rate varies slightly at different values of major cycle $\Delta K$, but in practice the exchange rate at a $K_{\max }$ of $20 \mathrm{MPa} \sqrt{m}$ is used throughout the life calculation.

If the peak stress in the loading cycle occurs at a temperature which is lower than the cycle maximum, the time independent crack growth is calculated by averaging the rates from the temperature associated with the peak cycle stress and the peak cycle temperature. 
In the $-180^{\circ}$ OOP tests considered in the current fractography study it is clear that time independent contributions dominate fatigue crack propagation life, since the maximum temperature occurs at the minimum stress. Subsequently a relatively slow crack growth rate is obtained. The OOP experiments performed at $-90^{\circ} \mathrm{CW}$ and ACW give very small time dependent contributions since the peak stress in the cycle occurs at the relatively low temperature of $500{ }^{\circ} \mathrm{C}$ and the stresses are low close to the peak cycle temperature. The IP tests performed at $0^{\circ}$ give large contributions from both time dependent and time independent crack growth, since the maximum stress occurs at the maximum temperature. As a consequence the crack propagation life is decreased due to interactions between fatigue and other damage mechanisms such as environmental degradation and creep.

The results of the model can be compared with the experimental data, Figure 11. Figure 11a shows a reasonable comparison between predictions and experimental data for a $-180^{\circ}$ OOP test performed at $\Delta \varepsilon=1.2 \%$, although the crack growth rates in the specimen do exceed the model predictions slightly. However, within the bounds of typical experimental scatter in crack propagation testing, this type of variation is not untypical. Further confidence from the model is gained from the predictions made of a $-90^{\circ}$ OOP test at $\Delta \varepsilon=1 \%$, Figure $11 \mathrm{~b}$, since a larger element of time dependence of the crack growth rate must be considered, with maximum stress occurring at $500{ }^{\circ} \mathrm{C}$. Clearly, further evaluation of the model is necessary, and since striation counting may be limited in scope in the current specimens, particularly IP specimens where creep/environment interactions make striation recognition difficult, this comparison will be required to be made with data from the TMF crack propagation facility currently under development.

Figure 11. TMF crack propagation predictions for (a) $-90^{\circ}$ OOP test at $\Delta \varepsilon=1 \%$; (b) $-180^{\circ}$ OOP test at $\Delta \varepsilon=1.2 \%$.

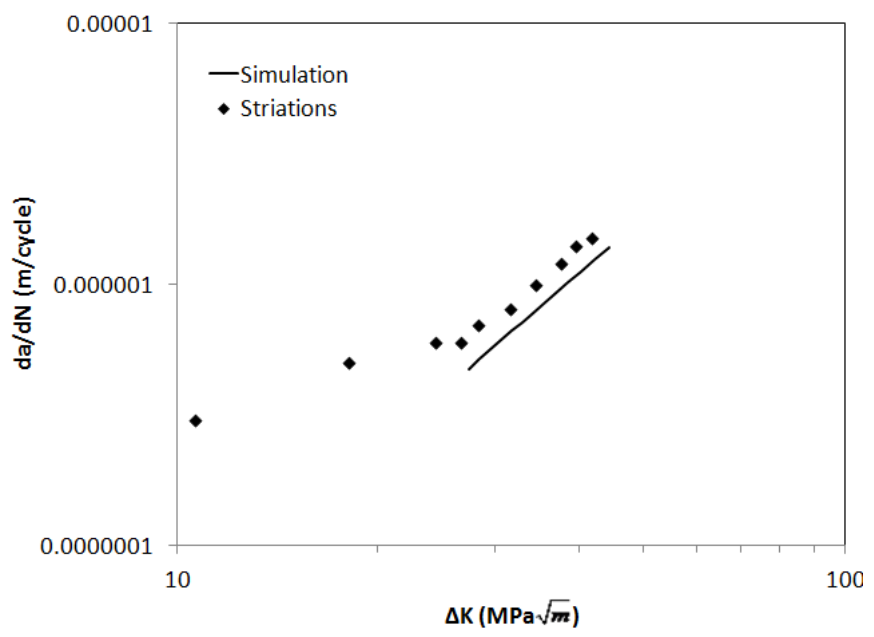

(a)

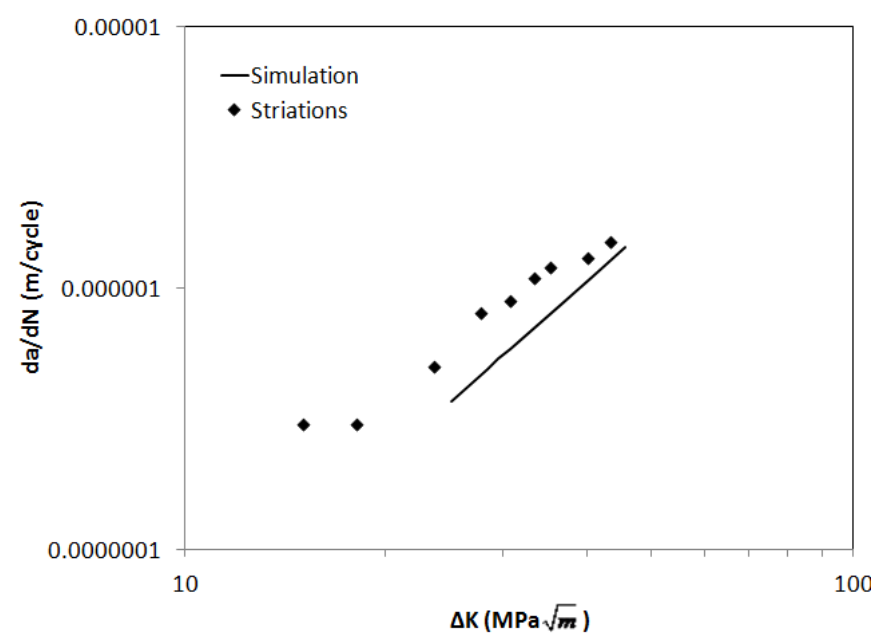

(b)

Each of the methods detailed above clearly have their own advantages and disadvantages. Fatigue life predictions based purely on mean stress provide reasonable ordering of results for a single strain range, but this approach is clearly limited for different strain ranges as the applied stress range varies. However, the trends indicate the importance of the interactions between creep and plasticity within the TMF tests, in particular the shape of the hysteresis loops produced and the maximum and minimum 
applied stresses. Subsequently the second approach involving the Basquin equation shows the dependence of TMF life on the stabilised stress range where a strong relationship is evident.

The Basquin equation derived for the current material clearly gives a reasonable methodology for prediction of alternative phase angles, based solely on the IP data set. However, it should be acknowledged that this relationship exists only over a limited range of TMF lives, with only two tests showing lives in excess of 3000 cycles. As shown by the striation counting exercise, these experiments are heavily influenced by crack propagation, with even the $-90^{\circ}$ OOP test at $\Delta \varepsilon=1 \%$ spending greater than $80 \%$ of its life in propagation. As peak strains and/or strain ranges are reduced however, this trend is unlikely to continue, with significant fractions of the total life spent initiating a crack, and as such the relationship is unlikely to remain as strong.

In order to overcome this issue it is necessary to consider a combination of methods. The generation of longer life TMF data would undoubtedly result in a shallower fatigue curve at extended lives, which when extrapolated to shorter lives would tend to underestimate the fatigue life response. However, proportionally the propagation life which would be insignificant at longer lives would become increasingly more important at shorter lives. As such a Basquin type approach with an added crack propagation phase is likely to produce the most appropriate methodology for lifing a wide variety of TMF experiments, particularly in the case of notched specimens where crack initiation is usually followed by a significant period of crack propagation.

\section{Conclusions}

Since the recognition of TMF as a significant life limiting factor, engineers have desired a methodology by which TMF behaviour can be accurately described by extrapolation of isothermal fatigue data, so that the wide range of possible parameters can be predicted without the need for large, expensive, protracted test programmes. The current work has described three methods by which predictions can be made. Under test conditions where a constant strain range is utilised across phase angles, it is shown that mean stress is the dominant factor and that a relationship exists between mean stress and fatigue life. This methodology, however, soon falters as the mechanical strain range is varied because of the resultant changes in the stabilised stress range. In this case it is demonstrated that a simple Basquin type equation can be effective as a life prediction technique, albeit for a range of TMF data which shows only short lives. It is acknowledged that such an approach is unlikely to extrapolate well to longer fatigue lives, because the proportionate difference in the ratio of crack initiation/propagation that is likely to occur. As such, it is concluded that a combination of a Basquin type approach based on longer term TMF data, supplemented by a crack propagation phase is the most likely method to provide accurate predictions over a range of test conditions.

Modelling approaches for both the initiation phase and propagation phase have been suggested, with the initiation phase based on the generation of hysteresis loops from an appropriate isothermal material database, from which a Basquin equation can be derived to allow a fatigue life prediction to be made. A crack propagation model which takes into account both time dependent and time independent crack growth has also been derived which shows good correlation to experimental crack propagation data derived from striation counting studies. 


\section{Acknowledgements}

The authors would like to acknowledge funding from the Engineering and Physical Sciences Research Council (EPSRC) and Rolls-Royce plc. in the undertaking of this research and in particular the contribution of Svjetlana Stekovic.

\section{Conflicts of Interest}

The authors declare no conflict of interest.

\section{References}

1. Christ, H.-J.; Jung, A.; Maier, H.J.; Teteruk, R. Thermomechanical fatigue-Damage mechanisms and mechanism-based life prediction methods. Sadhana 2003, 28, 147-165.

2. Sehitoglu, H.; Maier, H.J. Thermo-Mechanical Fatigue Behaviour of Materials; ASTM International: West Conshohocken, PA, USA, 2000.

3. Sakaguchi, M.; Okazaki, M. Thermo-mechanical and low cycle fatigues of single crystal Ni-base superalloys; Importance of microstructure for life prediction. JSME Int. J. Ser. 2006, 49, 345-354.

4. Pernot, J.J.; Nicholas, T.; Mall, S. Modelling thermomechanical fatigue crack growth rates in Ti-24Al-11Nb. Int. J. Fatigue 1994, 16, 111-112.

5. Liu, F.; Ai, S.H.; Wang, Y.C.; Zhang, H.; Wang, Z.G. Thermal-mechanical fatigue behaviour of a cast K417 nickel-based superalloy. Int. J. Fatigue 2002, 24, 841-846.

6. Coffin, L.F. Fatigue at Elevated Temperatures; ASTM International: Connecticut, CT, USA, 1973; pp. 744-782.

7. Manson, S.S. Fatigue: A complex subject-Some simple approximations. Exp. Mech. 1965, 5, 193-226.

8. Okazaki, M.; Sakaguchi, M. Thermo-mechanical fatigue failure of a single crystal Ni-based superalloy. Int. J. Fatigue 2008, 30, 318-323.

9. Zhang, J.X.; Harada, H.; Koizumi, Y.; Kobayashi, T. Crack appearance of single-crystal nickel-base superalloys after thermomechanical fatigue failure. Scr. Mater. 2009, 61, 1105-1108.

10. Henderson, P.J.; Sandström, R.; Ivarsson, B.G. Thermo-mechanical fatigue of the austenitic steel 253MA. Appl. Stainl. Steels 1992, 1, 94-103.

11. Kang, H.; Lee, Y.; Chen, J.; Fan, J.D. Thermo-mechanical fatigue damage model for variable temperature and loading amplitude conditions. Int. J. Fatigue 2007, 29, 1797-1802.

12. Okazaki, M.; Koizumi, T. Relationship of crack growth between thermal-mechanical and isothermal low-cycle fatigue at elevated temperatures. J. Eng. Mater. Technol. 1987, 109, 114-118.

13. Jordan, E.H.; Meyers, G.J. Fracture mechanics applied to nonisothermal fatigue crack growth. Eng. Fract. Mech. 1986, 23, 345-358.

14. ISO 12111:2011. Metallic Materials-Fatigue Testing-Strain-Controlled Thermo Mechanical Fatigue Testing Method; International Organization for Standardization: Geneva, Switzerland, 2011.

15. Hahner, P.; Affeldt, E.; Beck, T.; Klingelhoffer, H.; Loveday, M.; Rinaldi, C. Validated Code-of-Practice for Strain-Controlled Thermo-Mechanical Fatigue Testing. Available online: http://www.tmf-workshop.bam.de/en/tmf_media/eur22281en.pdf (accessed on 12 November 2013). 
16. Walker, K. Effects of environment and complex loading history on fatigue life. ASTM Int. 1970, $462,1-14$.

17. Basquin, O.H. The exponential law of endurance tests. ASTM 1919, 10, 625-630.

18. Evans, W.J.; Screech, J.E.; Williams, S.J. Thermo-mechanical fatigue and fracture of INCO718. Int. J. Fatigue 2008, 30, 257-267.

19. Mroz, Z. An attempt to describe the behaviour of metals under cyclic loads using a more general workhardening model. Acta Mech. 1967, 7, 199-212.

20. Lancaster, R.J.; Whittaker, M.T.; Williams, S.J. A review of thermo-mechanical fatigue behaviour in polycrystalline nickel superalloys for turbine disc applications. Mater. High Temp. 2013, 30, 2-12.

21. Jacobsson, L.; Persson, C.; Melin, S. Thermo-mechanical fatigue crack propagation experiments in Inconel 718. Int. J. Fatigue 2009, 31, 1318-1326.

22. Jacobsson, L.; Persson, C.; Melin, S. In-situ ESEM study of thermo-mechanical fatigue crack propagation. Mater. Sci. Eng. 2008, 496, 200-208.

23. Jacobsson, L.; Persson, C.; Melin, S. Experimental Methods for Thermomechanical Fatigue in Gas Turbine Materials. In Proceedings of the 15th European Conference of Fracture, Stockholm, Sweden, 11-13 August 2004.

24. Moverare, J.J.; Gustafsson, D. Hold-time effect on the thermo-mechanical fatigue crack growth behaviour of Inconel 718. Mater. Sci. Eng. 2011, 528, 8660-8670.

25. Magadanz, C.M. Effect of machining damage on low cycle fatigue crack initiation life in drilled holes in Udimet(RTM) 720. Master's Thesis, Purdue University Lafayette, IN, USA, November 2012.

26. Garcia de la Yedra, A.; Martin-Meizoso, A.; Rodiguez Martin, R.; Pedrejon, J.L. Thermo-mechanical fatigue behaviour and life prediction of C-1023 nickel based superalloy. Int. J. Eng. Sci. Technol. 2011, 3, 88-101.

27. Pineau, A.; Antolovich, S.D. High temperature fatigue of nickel-based superalloys - A review with special emphasis on deformation modes and oxidation. Eng. Fail. Anal. 2009, 16, 2668-2697.

(C) 2013 by the authors; licensee MDPI, Basel, Switzerland. This article is an open access article distributed under the terms and conditions of the Creative Commons Attribution license (http://creativecommons.org/licenses/by/3.0/ 